\title{
Does Long-Term Playing Violin Cause Unilateral Hearing Loss?
}

\author{
Özlem KONUKSEVEN ${ }^{1}$, Merve MERAL ${ }^{1}$, Ayşe Nur KARA ${ }^{1}$, Melis \\ ÖZDEMIR ${ }^{1}$, Gülnaz TANDOĞRU ${ }^{1}$
}

\begin{abstract}
Objective: This study compared the differences between two ears, the one which is close to the violin and the far one from the violin, in individuals who have been playing violin, and evaluated by using Distortion Product Otoacoustic Emission.

Methods: 30 individuals between the ages of 18-30, playing violin for at least 2 hours a day for 5 years and having no history of hearing loss or ear diseases were included in the study. Pure tone audiometry test and Distortion Product Otoacoustic Emission test were apLONplied on all subjects included in the study. The differences in these measurements for between two ears were subjected to statistical analyses.

Results: In all frequencies $(996-7998 \mathrm{~Hz})$ SNRs of the ear close to the violin and the ear far from the violin were obtained normal, above $6 \mathrm{~dB}$ SNR in the results of Distortion Product Otoacoustic Emission. However, there were not statistically significant differences between two ears ( $p>0.05)$, only at $1416 \mathrm{~Hz}$ a statistically significant difference was observed between the two ears $(p=0.03)$.

Conclusion: The violinists had better hearing function than expected. But noiseinduced hearing loss happens slowly over time and may be hard to detect at first. Violinists are at risk for occupational exposure to high noise. Therefore, the violinists with normal hearing should be monitored by DPOAE which shows noise-induced damage in outer hear cells.
\end{abstract}

Keywords: audiometry, otoacoustic emission, noise-induced hearing loss, violin, sound exposure

1 Department of Audiology, Faculty of Health Sciences, Istanbul Aydin University, Istanbul, Turkey

DOI: 10.17932/EJOH.2020.022/ejoh_v01i1004 


\section{Introduction}

High noise level causes mechanical damage in the inner ear, usually causing biochemical and cell changes in the outer hair cells and cell damage in the nerve fibers. As a result, temporary or permanent noise-induced hearing loss may occur $(1,2)$. Prolonged exposure to low noise levels may have the same effect as short exposure to high noise levels. For example, listening to a $100 \mathrm{~dB}$ sound for 15 minutes will have the same effect as listening to a sound of $85 \mathrm{~dB}$ for 8 hours (3). Noise-induced hearing loss is the most common occupational disease in the USA. The average of $16 \%$ of adult hearing losses around worldwide are caused by occupational exposure to high noise (4).

The function of outer hair cells damaged by noise is evaluated by the otoacoustic emission test. With the otoacoustic emission test, very small intensity sound waves originating from the outer hair cells in the cochlea are recorded with a sensitive microphone placed in the external ear canal. There are two types of otoacoustic emissions used for clinical testing: transient evoked otoacoustic emissions (TEOAEs) and distortion product otoacoustic emissions (DPOAEs). DPOAEs are used to obtain frequency-specific data regarding cochlear sensitivity (5). For DPOAE measurements, the signal-to-noise ratio (SNR), which is a measure of the level of DPOAEs, should be $6 \mathrm{~dB}$ and above. Studies have demonstrated that noise exposure can lead decrease results of DPOAEs $(6,7)$.

Since musical instruments produce very loud sounds, they may cause hearing loss. Musicians are averagely exposed to sounds of 79-98 dB intensity during concerts and rehearsals. This intensity is in the range of 85-105 dBA in individuals who play the violin, which is the most well-known instrument of the stringed instrument family (8). Violin's fundamental frequencies are $196-3136 \mathrm{~Hz}$, and its harmonics are in the range $4-15 \mathrm{kHz}$. While playing the violin, the intensity of the sound emanating from the instrument is harmful for the ear which is close to the violin (9). It was observed that the left ear of musicians using string instruments was exposed to an average of $4.6 \mathrm{dBA}$ sounder than their right ears (8).

The aim of this study was to compare the differences between two ears, close to violin and the far one from the violin, in individuals who have been playing violin and evaluated by using DPOAE.

\section{Material and Methods}

The study was conducted in Istanbul Aydin University Faculty of Health Sciences Audiology Laboratory between April and May 2019. It was approved by the Istanbul Aydin University Non-Interventional Clinical Research Ethics Committee (registration number 2019/77) on April $25^{\text {th }}, 2019.30$ individuals, 5 males and 25 females, between the ages of 18-30 were included in the study. 
Inclusion criteria were determined as playing the violin for at least 2 hours a day for 5 years and having no history of hearing loss or ear diseases. External auditory canal pathologies, perforation in the eardrum, congenital ear anomalies are defined as an exclusion criteria Otoscopic examination was performed on all subjects included in the study, and Pure tone audiometry (PTA) test and DPOAE test were applied. DPOAE results were compared between the ear close to violin and the far one from the violin.

Audiometric evaluation

PTA was performed with Otometrics Madsen Astera model device. It was performed following audiometric evaluation procedures in a standard silent cabin complying with ANSI standards. Air conduction evaluations has been performed using Telephonic TDH-39 (Telephonics, USA) headphones in the range of 0.25-8 $\mathrm{kHz}$, and bone conduction evaluations using a Radioear B-71 (Radioear, USA) brand bone conduction vibrator in the range of 0.5-4 kHz. Pure tone avarege (PTA) were calculated by using the hearing thresholds at frequencies of 500, 1000, $2000 \mathrm{~Hz}$, and Godmann's classification (0 -25 dB HL normal hearing, 26 $-40 \mathrm{~dB}$ HL very mild hearing loss, $41-55 \mathrm{~dB}$ HL mild hearing loss, $56-70 \mathrm{~dB} \mathrm{HL}$ moderate hearing loss, $71-90 \mathrm{~dB}$ HL severe hearing loss, 91 and above $\mathrm{dB} \mathrm{HL}$ very severe hearing loss) for both ears.

Otoacoustic Emission Test

DPOAE test was evaluated using Otometrics Madsen Capella model device and a bandwidth in the range of $1-8 \mathrm{kHz}$ was selected. $65 \mathrm{~dB}$ SPL at Ll and $55 \mathrm{~dB}$ SPL at L2 were used. Two pure tones (F1 and F2) were used as acoustic stimuli. The SNR value was used as $6 \mathrm{~dB}$ intensity. DPOAE test results were evaluated according to the SNR value at 13 different frequencies (996, 1191, 1416, 1679, 2001, 2382, 2832, 3359, 4003, 4755, 5654, 6728, $7998 \mathrm{~Hz}$ ).

Statistical Analysis

Whether there is a difference between the results of the ear close to violin and the far one from the violin is tested with Paired $\mathrm{T}$ test for the samples which has normal distribution, and with Wilcoxon Signed Ranks Test for the samples which has not normal distribution. A value of $p<0.05$ was considered the limit of significance. The normality of the distribution will be tested with the ShapiroWilk test. The homogeneity of variances will be tested with Levene Test. In cases where the data are normally distributed and the variances are homogeneous, the Wilcoxon Signed Ranks test will be applied. SPSS 22.0 package program will be used for data analysis. 


\section{Results}

This study examined 30 individuals ( 25 females and 5 males), aged between 18 and $30(22 \pm 2,19)$ years old (mean $24,10 \pm 5,46)$. One subject held the violin close to their right ear, and $29(96.7 \%)$ held the violin close to their left ear. As analyzing the anamnesis taken 11 individuals (36.7\%) had tinnitus and 19 individuals $(63.3 \%)$ had no tinnitus; one $(3.3 \%)$ The individuals included in the study have been playing the violin for $7.47 \pm 2.67$ years, with an average of $4.07 \pm 1.43$ hours a day. As the result of the pure tone audiometry test, the degrees of hearing of 30 individuals were determined normal. There were not statistically significant differences between two ears ( $p>0.05)$. Individuals' pure tone thresholds of the ears close to the violin and the ears far from the violin are shown on the Figure 1.

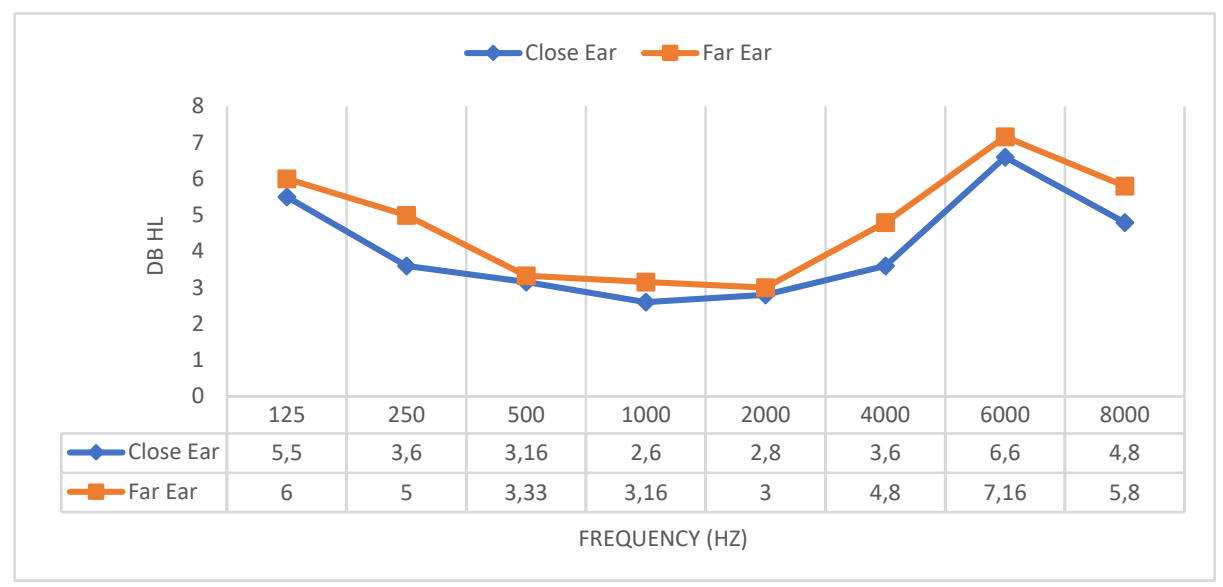

Figure 1: Pure tone threshold means of the ear close to the violin and the ear far from the violin

As the result of the DPOAE test, SNR of 30 individuals was compared. Since SNR values at $996 \mathrm{~Hz}$ data were not normally distributed, they were compared with the Wilcoxon Signed Ranks test. SNR values at $1191 \mathrm{~Hz}, 1416 \mathrm{~Hz}, 1680$ Hz, 2002 Hz, 2383 Hz, 2832 Hz, 3359 Hz, 4003 Hz, 4756 Hz, 5654 Hz, 6729 Hz $7998 \mathrm{~Hz}$ were compared with the Paired T test. In all frequencies, SNRs of the ear close to violin and the ear far from the violin were determined above $6 \mathrm{~dB}$ (Table 1). However, a statistically significant difference was observed between the two ears at $1416 \mathrm{~Hz}(\mathrm{p}=0.03)$. 
Table 1: SNR comparison of DPOAE of ears close and far from the violin

\begin{tabular}{cccccc}
\hline \multirow{2}{*}{ Frequency } & \multicolumn{2}{c}{ Close Ear } & \multicolumn{2}{c}{ Far Ear } & Sig (2-tailed) \\
& Mean \pm Sd & Min - Max & Mean \pm Sd & Min - Max & \\
\hline $996 \mathrm{~Hz}$ & $15.53 \pm 6.60$ & $7-27$ & $16.36 \pm 6.15$ & $6-27$ &, 437 \\
$1191 \mathrm{~Hz}$ & $17.63 \pm 6.91$ & $6-32$ & $17.70 \pm 7.95$ & $1-31$ &, 961 \\
$\mathbf{1 4 1 6 ~ H z}$ & $\mathbf{1 6 . 4 0} \pm \mathbf{6 , 5 4}$ & $\mathbf{6 - 3 0}$ & $\mathbf{1 8 . 6 0} \pm \mathbf{5 . 7 8}$ & $\mathbf{8}-\mathbf{2 8}$ &, $\mathbf{0 3 1 *}$ \\
$1679 \mathrm{~Hz}$ & $16.93 \pm 6.69$ & $3-30$ & $16.57 \pm 6.59$ & $0-27$ &, 692 \\
$2001 \mathrm{~Hz}$ & $15.33 \pm 6.67$ & $0-30$ & $14.06 \pm 5.92$ & $-1-25$ &, 381 \\
$2382 \mathrm{~Hz}$ & $12.53 \pm 4.28$ & $3-24$ & $11.8 \pm 4.77$ & $-4-22$ &, 758 \\
$2832 \mathrm{~Hz}$ & $13.00 \pm 5.03$ & $3-24$ & $12.26 \pm 5.50$ & $0-23$ &, 824 \\
$3359 \mathrm{~Hz}$ & $15.56 \pm 5.07$ & $8-24$ & $16.8 \pm 5.46$ & $7-27$ &, 248 \\
$4003 \mathrm{~Hz}$ & $14.46 \pm 4.25$ & $8-23$ & $13.9 \pm 5.98$ & $-2-26$ &, 703 \\
$4755 \mathrm{~Hz}$ & $11.83 \pm 6.35$ & $-3-21$ & $11.46 \pm 6.23$ & $-9-29$ &, 831 \\
$5654 \mathrm{~Hz}$ & $12.23 \pm 5.88$ & $1-26$ & $11.03 \pm 6.55$ & $-2-23$ &, 427 \\
$6728 \mathrm{~Hz}$ & $12.86 \pm 5.89$ & $-5-24$ & $10.96 \pm 7.00$ & $-9-27$ &, 294 \\
$7998 \mathrm{~Hz}$ & $8.33 \pm 4.47$ & $-4-18$ & $8.5 \pm 7.28$ & $-7-19$ &, 890 \\
\hline
\end{tabular}

\section{Discussion}

The presence of unilateral hearing loss in individuals exposed to violin noise for a long time was investigated in this study. The SNR value differences between two ears, close to the violin and the far one from the violin were compared by DPOAE at each frequency in the range of $996-7998 \mathrm{~Hz}$.

In the literature there are studies about the evaluation of hearing loss due to the noise in musicians. In a research paper, investigated the presence of noise-induced hearing loss in individuals playing violin. In the study, individuals playing violin were evaluated just before and after playing the violin, temporary hearing loss was found at $4 \mathrm{kHz}$ on left ear both at pure tone threshold and TEOAE tests (8). Schmidt et al. (8) were obtained that first violinists had a slightly worse hearing on the left ear compared with the right ear, for high frequencies. In our study, hearing thresholds and DPOAE results were normal in both ears. However, statistically significant difference was observed between the two ears at 1416 $\mathrm{Hz}$ of DPOAE while violinist PTA at $1416 \mathrm{~Hz}$ was normal. At the basis of the 
knowledge that is violin's fundamental frequencies are between $196-3136 \mathrm{~Hz}$, this may be explained violin's should be monitored with via DPOAE even if PTAs are normal. On the other hand, another explanation of this result may be that audiological test results were considered to be normal since individuals were not evaluated immediately after noise exposure or permanent hearing loss has not yet occurred as it causes temporary hearing loss.

The OSHA hearing conservation amendment mandates audiometric surveillance of workers who are exposed to noise levels equal to or exceeding $85 \mathrm{dBA}$ on an 8 -hour time-weighted average (10). There are different results in the literature regarding the sound level which violinists are exposed. Schmidt et al. (11) determined that there was exposure to $95-96 \mathrm{~dB}$ in the left ear and 89-92 dB in the right ear. In another study the ranges of average sound levels were $86-93 \mathrm{~dB}(\mathrm{~A})$ for violins and violas (12). Playing the violin for 2 hours a day, which is our inclusion criteria, may not be sufficient hour to cause hearing loss. Occupational standards specify a maximum allowable daily noise dose, expressed in percentages. For example, a person exposed to $85 \mathrm{dBA}$ per NIOSH or $90 \mathrm{dBA}$ per OSHA over an 8 -hour work shift, will reach $100 \%$ of their daily noise dose. $\% 100$ noise dose for 2 hours is $91 \mathrm{dBA}$ per NIOSH or $100 \mathrm{dBA}$ per $\operatorname{OSHA}(13,14)$.

In conclusion, the violinists had better hearing function than expected. But noiseinduced hearing loss happens slowly over time and may be hard to detect at first. Violinists are at risk for occupational exposure to high noise. Therefore, violinists with normal hearing should be monitored by DPOAE which shows noise-induced damage in outer hear cells.

This study was limited by the relatively small sample size. A larger sample size might have led to significant differences. In addition, while evaluating hearing loss, the sound exposure of violin should be determined in both ears. Future studies should examine hearing function by classifying violin players by work experience (in years) and the number of normal working hours per week.

\section{References}

1. Dinh, C. T., Goncalves, S., Bas, E., Van De Water, T. R., Zine, A. (2015). Molecular regulation of auditory hair cell death and approaches to protect sensory receptor cells and/or stimulate repair following acoustic trauma. Front Cell Neurosci, 9: 96.

2. Turcot, A., Girard, S. A., Courteau M, Baril, J., Larocque, R. (2015). Noise-induced hearing loss and combined noise and vibration exposure. Occup Med, 3: 238-244.

3. World Health Organization. (2019). WHO-ITU global standard for safe listening devices and systems. https://apps.who.int/iris/bitstream/hand 
le/10665/280085/9789241515276-eng.pdf?ua=1. Acces: 25.08 .2020

4. Nelson, D. I., Nelson, R. Y., Concha-Barrientos, M., \& Fingerhut, M. (2005). The global burden of occupational noise-induced hearing loss. American Journal of Industrial Medicine, 48(6), 446-458. https://doi. org/10.1002/ajim.20223

5. Kathleen, C. M. C., James, E. P. (2012). Audiology, in: K.J. Lee (Ed.), K.J. Lee's Essential Otolaryngology Head and Neck Surgery, tenth ed., The McGraw-Hill Companies, pp. 49-51

6. Lapsley, Miller, J. A., Marshall, L. \& Heller, L. M. (2004). A longitudinal study of changes in evoked otoacoustic emissions and pure-tone thresholds as measured in a hearing conservation program. Int J Audiol , 43, 307-322.

7. Lapsley, Miller, J. A., Marshall, L., Heller, L. M. \& Hughes, L. M. (2006). Lowlevel otoacoustic emissions may predict susceptibility to noise-induced hearing loss. J Acoust Soc Am, 280 - 296.

8. Schmidt, J. H., Pedersen, E. R., Paarup, H. M., Christensen-Dalsgaard, J., Andersen, T., Poulsen, T., \& Bælum, J. (2014). Hearing loss in relation to sound exposure of professional symphony orchestra musicians. Ear and Hearing, 35(4), 448-460. https://doi.org/10.1097/ AUD.0000000000000029

9. Chasin, M. (2012). Music and hearing aids. Trends in Amplification 16(3) 136-139.

10. Hallmo, P., Borchgrevink, H. M. \& Mair, I. W. (1995). Extended highfrequency thresholds in noise-induced hearing loss. Scand Audiol, 24, 47-52.

11. Schmidt, J. H., Pedersen, E. R., Juhl, P. M. (2011). Sound exposure of symphony orchestra musicians. Ann Occup Hyg. 55, 893-905.

12. Royster, J. D., Royster, L. H., \& Killion, M. C. (1991). Sound exposures and hearing thresholds of symphony orchestra musicians. The Journal of the Acoustical Society of America, 89 (6), 2793

13. National Institute for Occupational Safety and Health (1998). Criteria for a recommended standard: Occupational noise exposure. U.S. Department of Health and Human Services, Centers for Disease Control and Prevention.

14. Occupational Safety and Health Administration (1983). 29CFR1910.95 Occupational Noise Exposure: Hearing Conservation Amendment. 